Suite aux progrès de la médecine et à la complexification des traitements, la prise en charge des patients nécessite l'intervention d'un nombre croissant de spécialistes et professionnels médicaux et non médicaux qui interviennent pendant une phase de maladie de manière séquentielle ou parallèle. En conséquence, une collaboration constructive des différentes disciplines impliquées est indispensable pour le bien du patient. La FMCH et la FMH soutiennent leurs organisations et leurs membres dans ce défi. Nous remercions tous ceux qui s'engagent dans ce sens activement à nos côtés.

Dr méd. Josef E. Brandenberg, président FMCH, Dr méd. Christoph Bosshard, vice-président FMH

\title{
Le processus de traitement périopératoire
}

\section{Sandra Becker, Sina Grape, Christoph Ellenberger, Christof Heim, Sebastian Krayer, Alex Noser, Christine Zehntnera}

Commission pour les questions de structure et de processus, Société Suisse d'Anesthésiologie et de Réanimation ( ${ }^{a}$ présidente). Sur mandat et recommandation de la direction de la SSAR

Les références se trouvent sous www.bullmed.ch $\rightarrow$ Numéro actuel ou $\rightarrow$ Archives $\rightarrow 2018 \rightarrow 42$.

Pour des raisons rédactionnelles et afin de faciliter la lisibilité, nous employons dans ce texte la forme masculine ou féminine pour les deux sexes. La version allemande fait foi.

Dans le processus de soins périopératoire, la collaboration s'avère être une tâche très exigeante, de nombreuses disciplines et secteurs professionnels se sentant à la fois obligés de défendre leurs intérêts et en même temps devant coordonner leurs actions. L'élaboration et la mise en œuvre de parcours de soins adaptés aux patients, qui soient utilisables dans la pratique journalière et qui respectent les dispositions légales, doivent être le devoir des hôpitaux individuels et des centres opératoires. Les associations professionnelles, $\mathrm{H}+$, les groupes de qualité, les cantons, la Confédération, etc. sont appelés - où cela est nécessaire - à définir les lignes directrices.

\section{La problématique}

Pour qu'un système de santé soit de qualité et avec une efficience et une efficacité prouvées dans la gestion du processus de soins, une collaboration étroite et dynamique entre les différents acteurs est nécessaire. Cette collaboration peut cependant s'avérer difficile en raison des besoins partiellement contradictoires des acteurs concernés. En plus, elle peut être sensiblement perturbée en raison de conditions structurelles déficientes. L'environnement complexe en salle d'opération est typiquement marqué par d'importantes différences dans la manière de penser, dans les fonctions et les attentes des différents acteurs. Si un déséquilibre existe entre les acteurs prenant part au processus de soins, si une profession devient (trop) dominante vis-à-vis des autres ou s'il existe un flou dans la répartition des tâches et des responsabilités, cela peut entraver la collaboration et l'efficacité du traitement et nuire de manière importante à la satisfaction au travail du personnel. Le respect mutuel des acteurs concernés du point de vue professionnel et personnel, une hiérarchie svelte, une communication directe et sincère, de la disponibilité, ainsi que la volonté d'agir de façon adaptée et flexible promeuvent non seule- ment la collaboration participative, mais en sont les conditions préalables.

La planification et la réalisation de gestes opératoires sont l'exemple type d'une collaboration interconnectée, interdisciplinaire et interprofessionnelle dans le processus de soins médicaux. Afin d'obtenir une trajectoire sans encombre dans le processus périopératoire depuis le premier contact jusqu'à la clôture du cas, différents spécialistes doivent se coordonner et collaborer étroitement. A part l'opérateur et l'anesthésiste, la participation d'autres corps de métier comme les techniciens en salle d'opération, les infirmiers anesthésistes, les gestionnaires de matériel, etc. en tant qu'équipe rodée joue un rôle décisif pour la réussite d'une intervention. S'ajoutent au cas par cas des services auxiliaires comme la radiologie, le laboratoire (dont l'hématologie et le service de transfusion) et éventuellement d'autres thérapeutes spécialisés (physiothérapeutes, ergothérapeutes...).

Des tâches et mesures de soins spécifiques sont planifiées simultanément ou l'une après l'autre par des acteurs différents sous leur propre responsabilité ou alors en impliquant une responsabilité collective. 
Comme toutes les personnes impliquées ont rarement en leur possession en même temps l'ensemble des informations concernant le processus de soins, la collaboration efficace des différents corps de métier dans un même but constitue une tâche exigeante. Afin de garantir l'échange d'informations, des concepts et processus de soins définis de manière multilatérale s'avèrent indispensables, tout comme des rapports et discussions de cas réguliers, des intervisions et des supervisions.

Aussi bien la déclaration "Sécurité en Chirurgie» [1] de la fondation Sécurité des Patients Suisse que la charte "Collaboration entre les professionnels de la santé» [2] de l'Académie Suisse des Sciences Médicales et la «Helsinki Declaration on Patient Safety in Anesthesiology» [3] mettent au point des recommandations quant aux actions nécessaires pour garantir et améliorer la qualité des soins en médecine. Une coordination optimale des activités promet un grand potentiel de synergie avec comme conséquences une amélioration du flux des patients et une plus grande productivité du groupe.

En raison de la structure hétérogène des différentes institutions (hôpitaux, cliniques, centres de chirurgie, cabinets), des intérêts partiellement divergents de groupes individuels et des différentes conduites à tenir des corps de métier concernés, l'idée de pouvoir définir un concept pour le processus de soins périopératoire qui soit simple, applicable à toutes les situations et universellement accepté n'est probablement pas réaliste.

Par contre, les institutions ayant un rôle chirurgical doivent, en raison de la responsabilité organisationnelle, régler les compétences entre leurs différents intervenants au niveau local. Un prérequis étant des directives "palpables» qui constituent le cadre professionnel; directives qui - selon nécessité - sont à définir en priorité à travers un consensus des associations professionnelles ayant une activité chirurgicale et invasive chapeautée par la FMCH. En conséquence, les institutions sont appelées à initier la réalisation de telles directives au sein des associations professionnelles concernées, des organisations faîtières ainsi qu'avec les regroupements de santé nationaux.

Dans la partie suivante, des exemples types de différentes mesures thérapeutiques et interventionnelles (faisant partie du processus de soins périopératoire) sont présentés, pour lesquels un consensus entre les différents acteurs (par ex. chirurgiens, anesthésistes) est indispensable.

\section{Phase préopératoire - évaluation ciblée avec appréciation du risque}

Le spécialiste d'une discipline chirurgicale et invasive assume la responsabilité primaire de la planification en tant qu'initiateur et souvent comme responsable du cas. En règle générale, il réalise lui-même seulement une partie des prescriptions et actions et repartit voire délègue le reste des tâches qui sont non spécifiques à sa discipline opératoire. Dans le cas où l'acte chirurgical est réalisé sous anesthésie, l'opérateur partage la responsabilité dans différents sous-domaines avec l'anesthésiste. Dans cette situation, divers aspects en relation avec l'intervention chirurgicale doivent être considérés et clarifiés au besoin:

- Planification coordonnée des rendez-vous préopératoires du patient et coordination interdisciplinaire des consultations, également par rapport aux contenus (anamnèse, examen physique, informations générales sur le déroulement du traitement et sur la préparation à l'opération, etc.);

- Information et obtention du consentement du patient (Informed Consent [4]), aussi bien pour la procédure chirurgicale que pour l'anesthésie;

- Etablissement d'examens standardisés valables pour certaines catégories de patients et d'interventions;

- Organisation des examens complémentaires au moment opportun (fonctions pulmonaires, échocardiographie, etc.), et - particulièrement pour les patients ayant des comorbidités sévères et par conséquent un risque élevé en lien avec le traitement chirurgical - optimisation du traitement de fond (par ex. traitement de l'hypertension artérielle, de la BPCO, etc.);

- Patient blood management (produits sanguins): estimation des pertes sanguines périopératoires et information appropriée au patient si le risque de saignement est élevé. L'indication d'examens supplémentaires ainsi qu'un traitement préopératoire d'une anémie doivent être rendus possibles dans les meilleurs délais [5]. Pour les patients qui refusent les produits sanguins (par ex. Témoins de Jéhova), des directives claires doivent être établies;

- Volonté du patient: il faut définir comment les souhaits exprimés par le patient (par ex. directives anticipées) peuvent être pris en compte et respectés en périopératoire.

En fonction des besoins, prévoir de coordonner l'inclusion d'autres groupes professionnels:

- Physiothérapie: par ex. pour la préhabilitation ou pour les exercices de marche préopératoires avec décharge des extrémités à l'aide de béquilles ou d'autres aides à la déambulation;

- Médecine intensive: information des patients concernant une éventuelle prise en charge postopératoire aux soins intensifs ou aux soins intermédiaires. 


\section{Phase intraopératoire - compétences} clairement établies

Durant la phase intraopératoire également, différents points sont à régler au niveau institutionnel:

\section{Responsabilité et compétences pour:}

- Radioprotection des patients et des collaborateurs en salle d'opération selon les directives de l'OFSP: gestion des dosimètres, formation obligatoire initiale puis continue du personnel exposé, etc. Entretien des appareils émettant des radiations et gestion des moyens de radioprotection;

- Etablissement et suivi des directives d'hygiène en salle d'opération: prévention des infections (par ex. désinfection des mains, désinfection de la zone opératoire). Nettoyage, désinfection et traitement des déchets. Mesures de protection et d'isolement en cas de germes transmissibles;

- Protection du personnel: sécurité au travail, conduite à tenir en cas de blessures par piqûre ou coupure, etc. (accident d'exposition au sang ou à des liquides biologiques).

\section{Emploi et utilisation de check-lists [1]}

Une des mesures centrales qui a prouvé son efficacité dans la réduction des risques lors de la préparation puis la réalisation d'un acte chirurgical est l'utilisation de check-lists. Des directives bien définies, une formation à leur utilisation et des répétitions régulières sont essentielles pour leur utilisation correcte. Les checklists permettent d'éviter spécifiquement les erreurs par inattention dans le cadre de la routine clinique et sont utiles du point de vue médico-légal.

3. Positionnement des patients pendant l'intervention En général, l'opérateur a besoin d'un positionnement spécifique du patient pour mener à bien l'intervention. Pour ce positionnement, les facteurs propres au patient et les intérêts des autres groupes professionnels doivent également être pris en compte. Des lésions dues au positionnement (lésions par compression des nerfs ou des tissus, ischémies des extrémités, etc.) doivent être évitées.

- Des standards locaux définissant le positionnement souhaité en fonction du type d'intervention devraient exister dans chaque institution;

- Les compétences pour leur mise en œuvre doivent être clarifiées;

- La réalisation et le contrôle du positionnement doivent être documentés;

- Les patients doivent être informés en temps utile sur les risques liés au positionnement et sur les éventuelles complications [6]. Ceci doit être documenté.

4. Concept de transfusion, gestion de la coagulation, hémovigilance

Dès que dans une institution, des actes chirurgicaux avec risque hémorragique accru sont réalisés ou que des patients avec troubles de la coagulation sont pris en charge, les aspects suivants sont à considérer:

- Un protocole décrivant la pratique de la transfusion et la gestion de la coagulation selon des lignes directrices valables est à établir. Celui-ci devra contenir un algorithme [7] en cas de saignements importants;

- En respectant des indications strictes et après avoir évalué les risques potentiels, une quantité suffisante de produits sanguins et de facteurs de coagulation doit être mise à disposition, en tenant compte des facteurs logistiques institutionnels (par ex. disponibilité de systèmes de récupération de sang autologue [cell saver], banque de sang, réserves de sang et logistique de transport pour les produits de transfusion et de coagulation labiles);

- Les compétences pour décider du moment et la façon de réaliser les transfusions [8] ainsi que les aspects de sécurité [9] dans le cadre de l'hémovigilance [10] doivent être établies.

5. Médication préalable et adjuvante: dosage, indication et moment d'administration

La gestion périopératoire des médicaments habituels des patients doit être établie en fonction des classes de substances. En particulier dans le cas d'une thérapie préexistante avec des anticoagulants et des inhibiteurs de l'agrégation plaquettaire, les valeurs cibles de coagulation visées au moment de l'opération doivent être discutées et coordonnées en prenant en considération le risque hémorragique spécifique à l'intervention et le risque thrombo-embolique du patient. Il est recommandé de décider de la procédure à suivre (poursuivre le traitement, mettre en pause, reverser, bridging) et de la responsabilité pour la gestion périopératoire en fonction de lignes directrices claires et contraignantes. Le domaine de la médication périopératoire englobe également les thèmes suivants:

- Directives pour la prophylaxie antithrombotique périopératoire;

- Directives réglant le moment adéquat pour l'administration de l'antibiothérapie périopératoire [11] pour la prophylaxie et la thérapie de l'infection;

- Gestion de la prophylaxie de l'endocardite lors de risques cardiaques spécifiques;

- Administration de médicaments parentéraux au moment adéquat (par ex. antifibrinolytiques, corticostéroïdes) selon les protocoles spécifiques au type d'intervention et à l'institution.

6. Garrots $[12,13]$

L'utilisation d'un garrot garantit des conditions chirurgicales optimales et des pertes sanguines minimales pour beaucoup d'opérations touchant les extrémités. A cause des risques potentiels liés à l'utilisation des gar- 
rots, en particulier lors de problèmes circulatoires préexistants, les acteurs doivent s'entretenir des éventuelles contre-indications et définir les limitations temporelles éventuelles:

- Définition de valeurs cibles réglant la pression maximale des garrots et la durée d'ischémie ininterrompue;

- Définition de la conduite à tenir lors de troubles préexistants de la circulation veineuse et/ou artérielle;

- Définition de la manière et du moment d'administration de la prophylaxie antibiotique périopératoire, par rapport au début et à la fin de la phase d'ischémie lors de l'indication à l'utilisation d'un garrot.

7. Sonde vésicale [14]

La pose périopératoire d'une sonde vésicale peut s'avérer nécessaire pour des raisons différentes. Les points à clarifier sont:

- Indication d'une vidange (ou d'un remplissage) de la vessie à court ou à plus long terme via une sonde transurétrale;

- Indication d'une vidange de la vessie suprapubienne (cystofix);

- Les directives pour l'hygiène et, le cas échéant, la nécessité d'une prophylaxie ou d'une thérapie antibiotique en cas de sonde vésicale in situ, en particulier lors d'interventions avec implantation de matériel prothétique (prothèses articulaires, interventions vasculaires et interventions sur les valves cardiaques).

\section{Gestion de la température [15]}

Une hypothermie $<35,5^{\circ} \mathrm{C}$ en périopératoire peut aussi engendrer des complications graves. Parmi ces dernières, on compte les arythmies cardiaques, les infarctus du myocarde, les troubles de la coagulation avec tendance accrue au saignement et besoin élevé en transfusion, les infections de plaie et les ulcères de décubitus. En conséquence, une hypothermie accidentelle peut influencer de façon décisive la qualité du résultat postopératoire.

- L'équipe en salle d'opération devrait être instruite concernant les risques d'une hypothermie chez le patient chirurgical. Une attention particulière est à porter également à l'hypothermie postopératoire;

- Des directives et des mesures contraignantes pour éviter l'hypothermie doivent exister et leurs effets doivent être vérifiés périodiquement.

raduction française par les Dresses Sina Grape et Céline Callias.

Correspondance:

SGAR/SSAR

Rabbentalstr. 83

CH-3013 Berne

info[at]sgar-ssar.ch peuvent réduire les risques de morbidité et de mortalité postopératoire. L'antalgie postopératoire se base sur les conditions suivantes:

- Evaluation régulière de la douleur et documentation standardisée de la valeur (échelles de la douleur) et des mesures correctrices;

- Etablissement de limites chiffrées pour la mise en route d'interventions;

- Elaboration de standards de traitement propres à l'institution (standard operating procedures, SOP) réglant le traitement de la douleur en postopératoire et, en particulier, des protocoles d'antalgie spécifiques aux différentes procédures chirurgicales;

- Elaboration de protocoles institutionnels pour la prophylaxie et le traitement des effets secondaires des antalgiques.

\section{Surveillance postopératoire [17]}

La surveillance dans la phase postopératoire immédiate est de la responsabilité conjointe de l'anesthésiste et de l'opérateur (ou du médecin responsable d'un acte diagnostique ou interventionnel). Elle nécessite les prérequis suivants:

- Une infrastructure adaptée à la surveillance postopératoire englobant la possibilité d'intervention en urgence en cas de complications cardiovasculaires, pulmonaires, neurologiques, gastroentérologiques et autres;

- Le personnel soignant est formé spécifiquement à la reconnaissance et au traitement des altérations des fonctions vitales, et peut prendre en charge de façon autonome les premiers soins lors d'évènements inattendus;

- Chaque hôpital ou centre opératoire doit établir des lignes directrices et des standards pour la surveillance postopératoire des patients. Font aussi partie de ces directives des critères de sortie contraignants après des interventions ambulatoires.

\section{Résumé}

La collaboration dans le cadre du processus thérapeutique périopératoire est un défi considérable au vu du nombre important de professionnels impliqués qui doivent faire coïncider leurs intérêts respectifs.

Des protocoles clairement définis et communiqués à tous permettent la transparence.

La prise de conscience de tous les participants, leur motivation et leur volonté d'avoir une collaboration constructive constituent les meilleures conditions pour offrir des traitements de qualité tout en limitant l'utilisation des ressources. Le feed-back positif et la satisfaction des patients ainsi que celle des personnes impliquées doivent nous motiver à poursuivre dans cette voie. 